\title{
Excited state charge transfer reaction in (mixed solvent + electrolyte) systems: Role of reactant-solvent and reactant-ion interactions
}

\author{
HARUN AL RASID GAZI and RANJIT BISWAS* \\ Department of Chemical, Biological and Macromolecular Sciences, S. N. Bose National Centre for Basic \\ Sciences, JD Block, Sector III, Salt Lake, Kolkata, 700 098, India \\ e-mail: ranjit@bose.res.in
}

MS received 2 September 2010; revised 31 January 2011; accepted 10 February 2011

\begin{abstract}
Fluorescence spectroscopic techniques have been used to study the excited state intramolecular charge transfer reaction of 4-(1-azetidinyl)benzonitrile (P4C) in two sets of mixed solvents, (1-propanol + ethyl acetate) and (propylene carbonate + acetonitrile), in the absence and presence of a strong electrolyte, lithium perchlorate. These two sets of mixed solvent systems represent binary solvent mixtures of low and high polarities, respectively. Density, sound velocity and viscosity measurements indicate that these two mixed solvent systems are structurally different. Stronger ion-reactant interaction is evidenced in the mole fraction independence of emission frequencies in electrolyte solutions of low polar binary solvent mixtures. For both these mixtures, the reaction driving force $\left(-\Delta G_{r}\right)$ decreases with increase in mole fraction of the relatively less polar solvent component of the mixture. Interestingly, $-\Delta G_{r}$ increases significantly on addition of electrolyte in low polar mixtures and exhibits mixture composition dependence but, in contrast, $-\Delta G_{r}$ in high polar mixtures does not sense variation in mixture composition in presence of electrolyte. This insensitivity to mixture composition for high polar mixtures is also observed for the measured reaction time constant. In addition, the reaction time constant does not sense the presence of electrolyte in the high polar solvent mixtures. The reaction time constant in low polar mixtures, which becomes faster on addition of electrolyte, lengthens on increasing the mole fraction of the relatively less polar solvent component of the mixture. These observations have been qualitatively explained in terms of the measured solvent reorganization energy and reaction driving force by using expressions from the classical theory of electron transfer reaction.
\end{abstract}

Keywords. Binary solvent mixture; low/high polar mixture; solvent reorganization energy; electrolyte solution.

\section{Introduction}

Electrolyte solutions of mixed solvents are important reaction media as one can tune the polarity of the solutions by altering either the mixture composition (in terms of mole fraction of solvent components) or by changing the concentration of a dissolved electrolyte. ${ }^{1-15}$ While modulation of solution polarity by changing mixture composition assists in product separation, addition of electrolyte is expected to facilitate formation of polar activated complex and product. In either of the cases, a chemical reaction involving activation barrier is considerably affected because of the polarity dependence of the barrier (solvent static effects), ${ }^{16-18}$ and any possible mismatch between the barrier crossing timescale and that of environment reorganization (or solvation) in response to the change in

\footnotetext{
${ }^{*}$ For correspondence
}

reactant (solvent dynamic effects). ${ }^{19,20}$ Since the solvent static and dynamic effects originate from the interaction of the reactant with the environment, study of medium effects provide understanding of the solutemedium interaction at the microscopic level. Eventhough a large number of study unravels the medium effects on a chemical reaction in pure solvents in the absence or presence of an electrolyte, ${ }^{21-28}$ similar study hardly exists for a reaction occurring in electrolyte solutions of mixed solvents. The complexity of the (mixed solvent + electrolyte) systems may be one of the reasons for such a rarity where both preferential solvation $^{29-31}$ and ion-reactant specific interaction can have profound effects on the outcome of a reaction. For example, while the solvent sorting by a polar reactant (preferential solvation) may partially reduce the reaction rate via slower diffusive solvent reorganization, this effect may become completely unimportant in presence of electrolyte due to ion-solute specific interaction. The interplay between preferential solvation and ion-solute 
Table 1. Solvent polarity parameters and field factors.

\begin{tabular}{lccccccc}
\hline Solvent & $\varepsilon_{0}$ & $\mathrm{n}$ & $\left(f \varepsilon_{0}\right)$ & $f\left(\mathbf{n}^{2}\right)$ & $\Delta f$ & $\mathrm{E}_{\mathrm{T}}(30)$ & $\pi^{*}$ \\
\hline Propylen carbonate & 64.92 & 1.42 & 0.96 & 0.25 & 0.71 & 46 & 0.83 \\
Acetonitrile & 35.94 & 1.342 & 0.92 & 0.21 & 0.71 & 45.6 & 0.66 \\
Propanol & 20.45 & 1.384 & 0.87 & 0.23 & 0.64 & 50.7 & 0.53 \\
Ethyl acetate & 6.02 & 1.37 & 0.63 & 0.23 & 0.40 & 38.1 & 0.45 \\
\hline
\end{tabular}

specific interaction is expected to be reflected both in the steady state spectral characteristics of a suitable reactant molecule and reaction rate constant of it in such mixtures.

In this paper, we present fluorescence spectroscopic results on reaction equilibrium and rate constants of the photo-excited intramolecular charge transfer reaction in 4-(1-azetidinyl)benzonitrile (P4C) in two types of mixed solvents in the absence and presence of an electrolyte at various mixture compositions. The chosen mixed solvent systems are (i) propylene carbonate + acetonitrile $(\mathrm{PC}+\mathrm{AN})$ and (ii) 1-propanol + ethyl acetate $(\mathrm{PrOH}+\mathrm{EA})$ mixtures. For each of these mixed solvent systems, the solvent components are miscible at all mole fractions at room temperature. Solvent characteristics summarized in table 1 indicate that these mixed solvent systems, based on the values of average static dielectric constant $\left(\varepsilon_{0}\right),{ }^{32}$ can be broadly divided into strongly and weakly polar mixed solvent systems. Values of the reaction field factors, ${ }^{33} f(x)=$ $[x-1] /[x+2]$ with $x=\varepsilon_{0}$ or $n^{2}$ ( $n$ being the refractive index) and their difference, $\Delta f=\left[\varepsilon_{0}-1\right] /\left[\varepsilon_{0}+\right.$ $2]-\left[n^{2}-1\right] /\left[n^{2}+2\right]$, indicate that propylene carbonate (PC) and acetonitrile (AN) are solvents of nearly equal polarities. This is further reflected in the very similar $E_{T}(30)$ values for these two solvents. ${ }^{34}$ Values in the $\pi^{*}$ polarity scale, ${ }^{35,36}$ on the other hand, seems to suggest that propanol $(\mathrm{PrOH})$ and ethyl acetate (EA) should be of similar polarities eventhough their $E_{T}(30), \Delta f$ and $f\left(\varepsilon_{0}\right)$ values are different from each other. Note in table 1 that $\pi^{*}$ values of these solvents follow the trend of $\varepsilon_{0}$ but not the $E_{T}(30)$ values. However, $\Delta f$ represents the polarity of these solvents in an over-all consistent manner and, therefore, grouping these binary solvent systems into strongly and weakly polar mixtures based on $\Delta f$ values appears logical.

The difference between these two mixed solvent systems does not lie only in their different polarity values. Data presented in figure 1 reveal that while density of $(\mathrm{PrOH}+\mathrm{EA})$ mixture increases with the increase in mole fraction of the relatively less polar solvent component (that is, EA), the reverse occurs for (PC + AN) mixtures, eventhough solution viscosity decreases for these mixtures on increase in the concentration of either EA or AN. The measured densities and viscosities in presence of electrolyte in these mixed solvents are given in table S1 (supporting information). Adiabatic sound velocity, measured at a single frequency and shown in figure 2, however, decreases with density for $(\mathrm{PrOH}+$ EA) mixtures but increases for (PC $+\mathrm{AN})$ mixtures. The decrease in both sound velocity and viscosity with
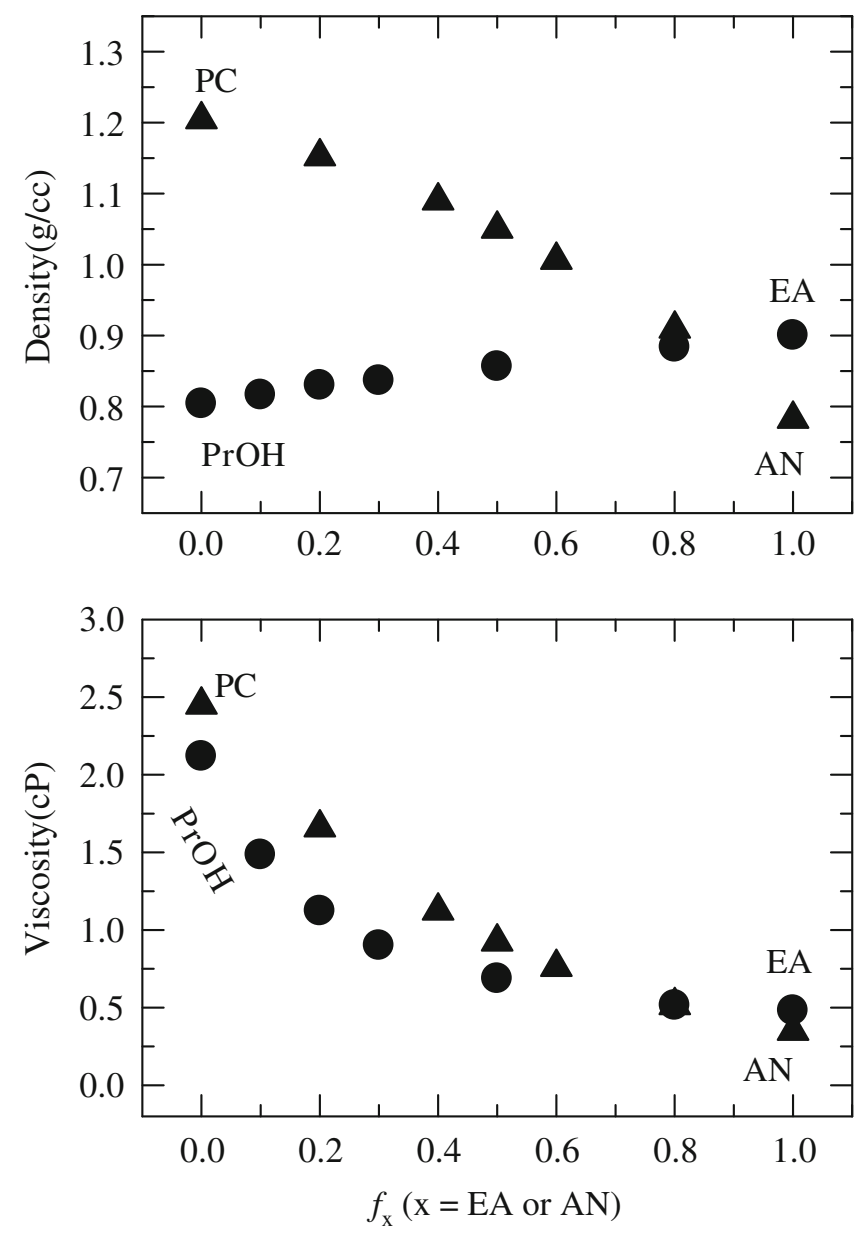

Figure 1. Mixture composition dependence of solution density and viscosity for $(\mathrm{PrOH}+\mathrm{EA})$ and $(\mathrm{PC}+\mathrm{AN})$ mixed solvent systems. Note that these quantities (measured) are shown as a function of the mole fraction of the relatively less polar solvent component constituting each of these different systems. 


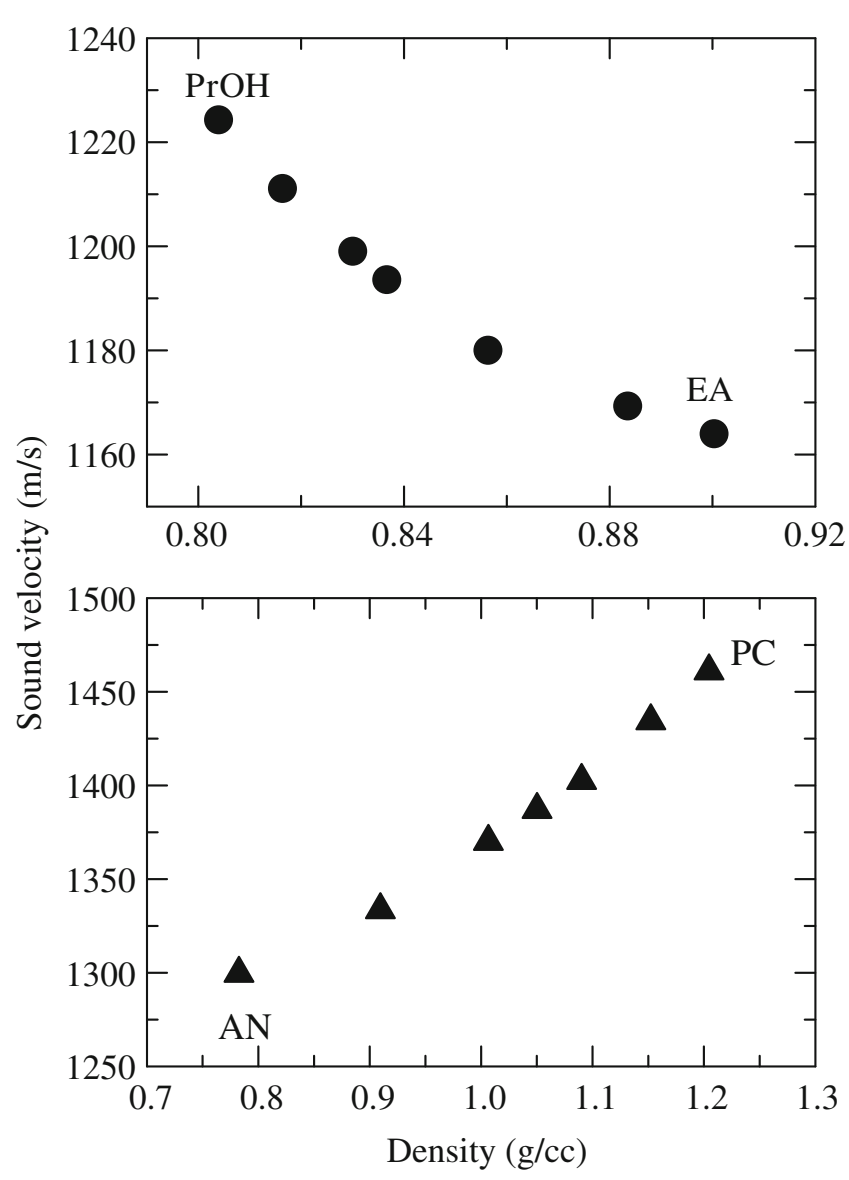

Figure 2. Density dependence of the measured sound velocity in $(\mathrm{PrOH}+\mathrm{EA})$ and $(\mathrm{PC}+\mathrm{AN})$ mixed solvent systems. Pure solvent ends are tagged with solvent abbreviations.

density for $(\mathrm{PrOH}+\mathrm{EA})$ mixture is therefore quite opposite to the hard sphere results ${ }^{37}$ and thus very different from the $(\mathrm{PC}+\mathrm{AN})$ mixed solvent system.

The concentration of $\mathrm{LiClO}_{4}$ has been kept fixed at $1.0 \mathrm{M}$ for $(\mathrm{PrOH}+\mathrm{EA})$ mixtures and $0.5 \mathrm{M}$ for $(\mathrm{PC}+\mathrm{AN})$ mixtures because larger concentrations of electrolyte than the specified ones become insoluble in one of the solvent components in each of these two mixed solvent systems. One can, in principle, carry out an electrolyte concentration dependence study for both the solutions at each mixture composition, but the main focus of the present study is to uncover the qualitative differences in solute-ion and solute-solvent interactions and their consequences on a simple chemical reaction occurring in these pair of mixed solvents of varying bulk polarity. Earlier measurements of reaction time scales of $\mathrm{P} 4 \mathrm{C}$ in neat polar solvents ${ }^{21}$ indicate that the broad time resolution employed in the present experiments would be able to describe semiquantitatively the effects of mixture composition and electrolyte on reaction time scale in this molecule. As before, ${ }^{21-28}$ we have used the twisted intramolecular charge transfer (TICT) model ${ }^{38,39}$ to explain the decay kinetics of P4C eventhough alternative models ${ }^{40,41}$ are available in the literature. Scheme 1 depicts the reaction in $\mathrm{P} 4 \mathrm{C}$ where, in the excited electronic surface $\left(\mathrm{S}_{1}\right)$, the photo-induced locally excited (LE) state converts to the relatively more polar charge transfer (CT) state with a forward reaction rate constant, $k_{f}$. Note that the $\mathrm{LE} \rightarrow \mathrm{CT}$ conversion reaction in $\mathrm{P} 4 \mathrm{C}$ is associated with an activation barrier ${ }^{21}$ of $\sim 6 k_{B} T$. Subsequently, the CT state either regenerates the LE state by participating in the reverse reaction with a rate constant, $k_{\mathrm{r}}$, or, decays to the ground electronic state $\left(\mathrm{S}_{0}\right)$ with net (radiative + non-radiative) rate constant, $k_{\mathrm{CT}}$. Depopulation from the photo-prepared LE state to the ground state can also take place through the net rate constant, $k_{\mathrm{LE}}$. We assume
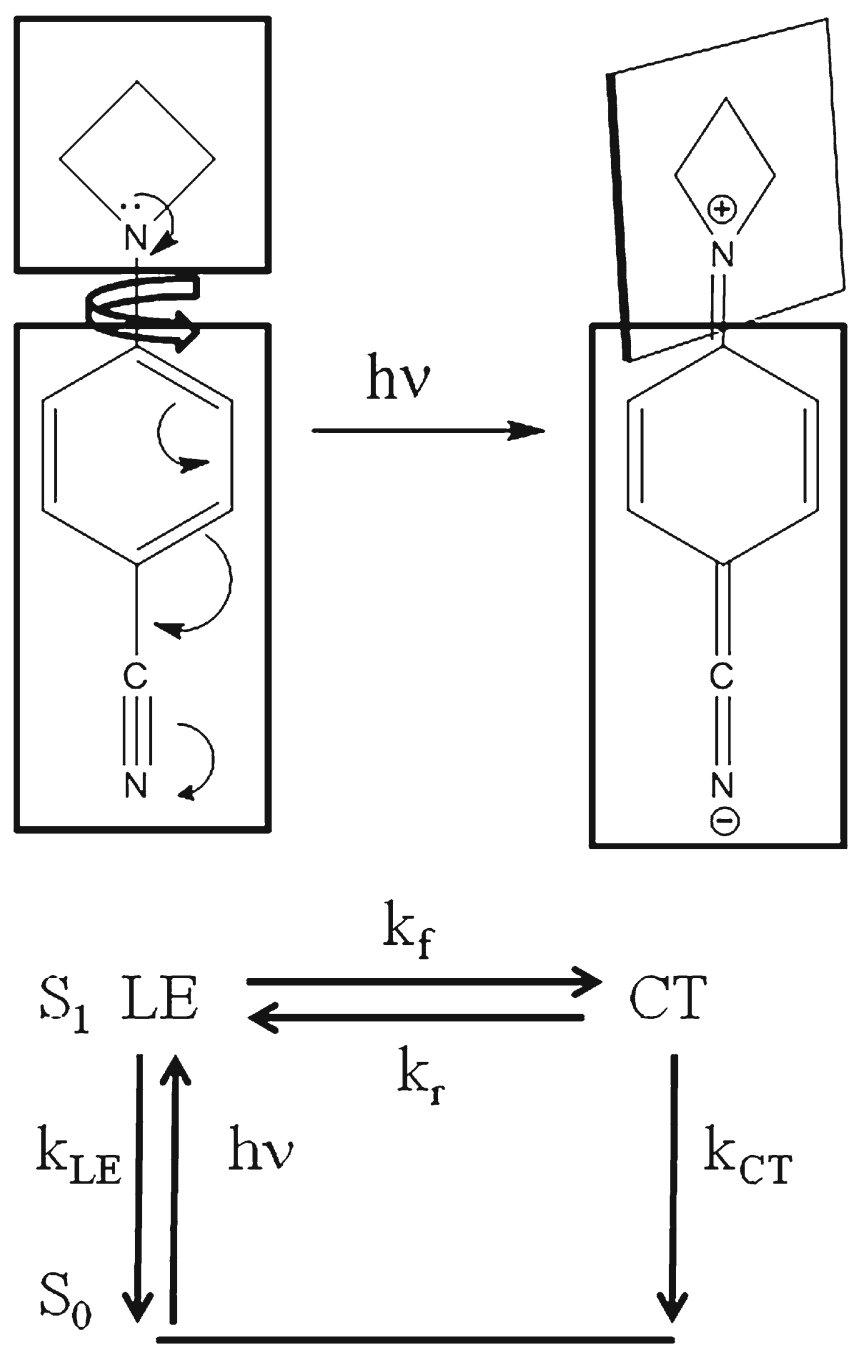

Scheme 1. Pictorial representation of photo-induced intramolecular charge transfer reaction in 4-(1-azetidinyl)benzonitrile $(\mathrm{P} 4 \mathrm{C})$ and the related kinetic model. 
that the reaction is in the rapid solvent equilibration limit and hence a biexponential decay of intensity with time is expected. The decay kinetics of $\mathrm{P} 4 \mathrm{C}$ is indeed bi-exponential in these binary solvents mixtures at all mole fractions both in the presence and absence of electrolyte. This again confirms the generality of two-state reversible reaction mechanism as found earlier for $\mathrm{P} 4 \mathrm{C}$ in neat polar solvents and electrolyte solutions in one component solvent. We use equations derived in ref. 21 to analyse the relevant spectroscopic data.

The organization of the rest of the paper is as follows. Experimental details are given in the section 2. Section 3 contains experimental results from our steady state and time resolved experiments. Supporting information is given wherever necessary. The concluding remarks are in section 4 .

\section{Experimental}

4-(1-Azetidinyl)benzonitrile (P4C) was synthesized by following method given in literature ${ }^{42,43}$ and recrystallized twice from cyclohexane (Merck, Germany). Purity of the compound was then checked by thin layer chromatography and monitoring the excitation wavelength dependence of the fluorescence emission.

Acetonitrile, ethyl acetate, propanol and propylene carbonate were used as received (spectrophotometric grade) from Aldrich. Lithium perchlorate $\left(\mathrm{LiClO}_{4}\right)$ was obtained (highest available grade) from Aldrich and vacuum dried prior to use. Coumarin 153 (C153) was from Exciton and used as received.

Binary mixtures of EA with PrOH, and AN with PC at several compositions were prepared by dissolving measured amounts of respective components at a given mixture composition. Subsequently, calculated amounts of $\mathrm{LiClO}_{4}$ were dissolved in order to prepare $1.0 \mathrm{M}$ solutions in $(\mathrm{PrOH}+\mathrm{EA})$ mixtures and $0.5 \mathrm{M}$ solutions in $(\mathrm{PC}+\mathrm{AN})$ mixtures at all compositions. The solution density, viscosity and ultrasound velocity were then measured by using an automated density-cumsound velocity analyzer (Anton Paar, model DSA 5000) at $293.15 \pm 0.5 \mathrm{~K}$ where the analyzer was first calibrated against the density of and sound velocity in air at that temperature. Fidelity of the measurements was then ensured via measuring these quantities for water and comparing with literature values. Subsequently, measurements for the solutions under study were carried out with solutions in a sequence of increasing concentration of the less polar solvent. Repeat experiments at a few points indicated the measured data are associated with $\pm 5 \%$ error of the reported values.
A fraction of these stock solutions was then taken into a quartz cuvette of optical path length $1 \mathrm{~cm}$. Before transferring the stock solution, 5 micro-litre of $\mathrm{P} 4 \mathrm{C}$ solution in heptane was taken in the cuvette and then a gentle stream of dry $\mathrm{N}_{2}$ gas was passed through to evaporate off the carrier non-polar solvent. Note that the concentration of $\mathrm{P} 4 \mathrm{C}$ was maintained at $\leq 10^{-5} \mathrm{M}$ in all binary mixtures both in the presence and absence of electrolyte studied here. The solution in the cuvette was then shaken very well and absorption spectrum was recorded using a UV-VIS absorption spectrophotometer (Shimadzu, UV-2450). Corrected fluorescence emission spectra were recorded using a fluorimeter (Jobin Yvon Horiba, Fluoromax-3) after exciting the sample at $300 \mathrm{~nm}$ for all measurements. Solvent blanks were subtracted from the emission spectra prior to analysis and converted to frequency representation after properly weighting the intensity with $\lambda^{2}$.

LE and CT band areas were then obtained after deconvolution of the full emission spectrum of $\mathrm{P} 4 \mathrm{C}$ into two fragments by shifting and broadening the emission spectrum of $\mathrm{P} 4 \mathrm{C}$ in perfluorohexane (reference emission spectrum). ${ }^{21}$ The linewidth of the individual bands (LE and CT) were determined by convoluting the reference emission with a Gaussian line broadening function (inhomogeneous solvent broadening assumed). A given experimental emission spectrum was then leastsquares fit to the model spectrum consisting of two bands derived from the shifted and broadened reference emission spectrum. As seen earlier, ${ }^{21}$ this method describes reasonably well both the LE and CT band shapes and allows determination of spectral properties with better accuracy. Emission peak frequencies were calculated from the shift of the emission spectra from the average peak frequency of the reference emission spectrum. The average peak frequency of the reference spectrum was calculated by simply averaging the number obtained by fitting the upper half of the spectrum with an inverted parabola, first moment and the arithmetic mean of the frequencies at half intensities on both the blue and red ends of the reference emission spectrum. ${ }^{44}$ Similar procedure, sans deconvolution, was used to obtain absorption peak frequencies from the solvent-background subtracted absorption spectra.

Time resolved emission data were collected employing time correlated single photon counting (TCSPC) technique based on a laser system (Edinburgh Instrument, Lifespec-ps) where light-emitting diode (LED) was the source for providing the excitation light at $299 \mathrm{~nm}$. The fwhm (full width at half maximum) of the instrument response function (IRF) with the above excitation was approximately 475 ps. The fluorescence 
emission decay was collected at magic angle at peak position of both LE and CT band of spectrum with a band-pass of $8 \mathrm{~nm}$. During analysis, the emission decays were first deconvoluted with the instrument response function to remove the effects of instrumental broadening. Subsequently, the deconvoluted decays were fitted to multi-exponential functions of time using an iterative reconvolution algorithm to finally arrive at the reaction time constants. It is to be mentioned here that such iterative reconvolution sharpens the effective time resolution by a factor of 3-5 over the IRF and thus enables one to capture much faster decay kinetics with reasonable accuracy. ${ }^{21,45,46}$ For all solutions, bi-exponential fit to each of the LE emission decays produced one short time constant and one long time constant, whereas the same for CT emission decays (collected wherever possible) generated a rise-time constant very similar to LE short time constant and one long time constant. Therefore, the short time constant associated with the LE decay was considered as the reaction time. The LE decays of the TICT molecules in non-polar solvents (heptane and hexane) were found to fit to single exponentials with only one long time constant. For a few cases, emission decays were collected at two or three different emission wavelengths around the LE and CT peaks and the analysed data were found to vary within a small uncertainty. Bubbling of dry argon gas through a few samples also produced either very insignificant or no effects in the analysed data.

\section{Results and discussion}

\subsection{Steady state spectral studies}

Representative absorption and emission spectra of $\mathrm{P} 4 \mathrm{C}$ in $(\mathrm{PrOH}+\mathrm{EA})$ and $(\mathrm{PC}+\mathrm{AN})$ binary mixtures at 0.2 , 0.5 and 0.8 mole fractions of less polar solvent component both in presence and absence of $\mathrm{LiClO}_{4}$ are shown in figure 3. It is clear from figure 3 that the amounts of blueshift in absorption spectra with increase in mole fraction of less polar solvent are larger for $(\mathrm{PrOH}+$ EA) binary mixtures both in the presence and absence of electrolyte than those in (PC $+\mathrm{AN})$ mixed solvent systems. Emission spectra also show the similar effects where the CT band blueshifts with simultaneous decrease in its area upon increasing the less polar solvent component in both the mixtures. Such an effect for the LE band is not so obvious because the shifts of the LE band is very small and well within the instrument resolution $\left( \pm 250 \mathrm{~cm}^{-1}\right)$. The fact that the effects of electrolyte are more pronounced in $(\mathrm{PrOH}+\mathrm{EA})$ mixtures than in $(\mathrm{PC}+\mathrm{AN})$ mixtures is more evident in figure 4 where the mole fraction dependent absorption and emission peak frequencies of $\mathrm{P} 4 \mathrm{C}$ in these mixtures, both in the presence and absence of electrolyte, are shown. Note in figure 4 that in the absence of electrolyte the total blueshift in absorption peak frequencies from pure to pure solvents is $\sim 400 \mathrm{~cm}^{-1}$ for $(\mathrm{PrOH}+$ EA) mixture and $\sim 200 \mathrm{~cm}^{-1}$ for (PC + AN) mixture eventhough these two mixed solvent systems differ (if compared between solvents of the lowest $\varepsilon_{0}$ values) in polarity at least by a factor of 2 . The CT emission band, which is associated with larger dipole moment than the ground electronic state, registers a blueshift of $\sim 1500 \mathrm{~cm}^{-1}$ for changing the mixture composition from pure $\mathrm{PrOH}$ to pure EA. In contrast, this is only $\sim 350 \mathrm{~cm}^{-1}$ for changing the solvent from $\mathrm{PC}$ to AN eventhough $\varepsilon_{0}$ values of these strongly polar solvents differ by nearly a factor of 2 . In fact, the insensitivity of steady state spectral properties to the mixture composition for $(\mathrm{PC}+\mathrm{AN})$ binary systems is already known ${ }^{33}$ and has been ascribed to the very similar $\Delta f$ values of these two solvents.

Data presented in figure 4 reveal that addition of electrolyte induces an extra stabilization of absorption frequency by $\leq 400 \mathrm{~cm}^{-1}$ in (PrOH $\left.+\mathrm{EA}\right)$ mixture for the whole composition range but the electrolyte-induced redshift of absorption frequency is limited to within $\sim 100 \mathrm{~cm}^{-1}$ for the (PC + AN) mixture across the mixture composition. Interestingly, the peak frequency of the CT emission band in presence of electrolyte remains insensitive to the addition of $\mathrm{EA}$ in $(\mathrm{PrOH}+$ EA) mixture for the entire composition eventhough the difference between the emission frequencies in the absence and presence of electrolyte, depending upon the mixture composition, varies between $\sim 1000 \mathrm{~cm}^{-1}$ and $2500 \mathrm{~cm}^{-1}$ in this binary system. For $(\mathrm{PC}+\mathrm{AN})$ mixture, in contrast, the CT band shows a very small redshift $\left(\sim 100 \mathrm{~cm}^{-1}\right)$ in presence of electrolyte for changing the mixture composition from PC to AN, and the electrolyte-induced stabilization ranges between $\sim 500 \mathrm{~cm}^{-1}$ and $800 \mathrm{~cm}^{-1}$. The relatively weaker effects of electrolyte on spectral properties in strongly polar media has also been observed earlier while studying Stokes' shift dynamics of non-reactive probes in electrolyte solutions, ${ }^{46}$ and also in electrolyte-induced modification of the equilibrium and reaction rate constants of TICT molecules in single component solvents of varying polarity. ${ }^{23-26}$ The weaker electrolyte effects may arise from the greater screening of the existing ions by the strongly polar medium which inhibits ionsolute direct interaction. A stronger ion-solvent interaction therefore leads to a weaker ion-solute interaction. 


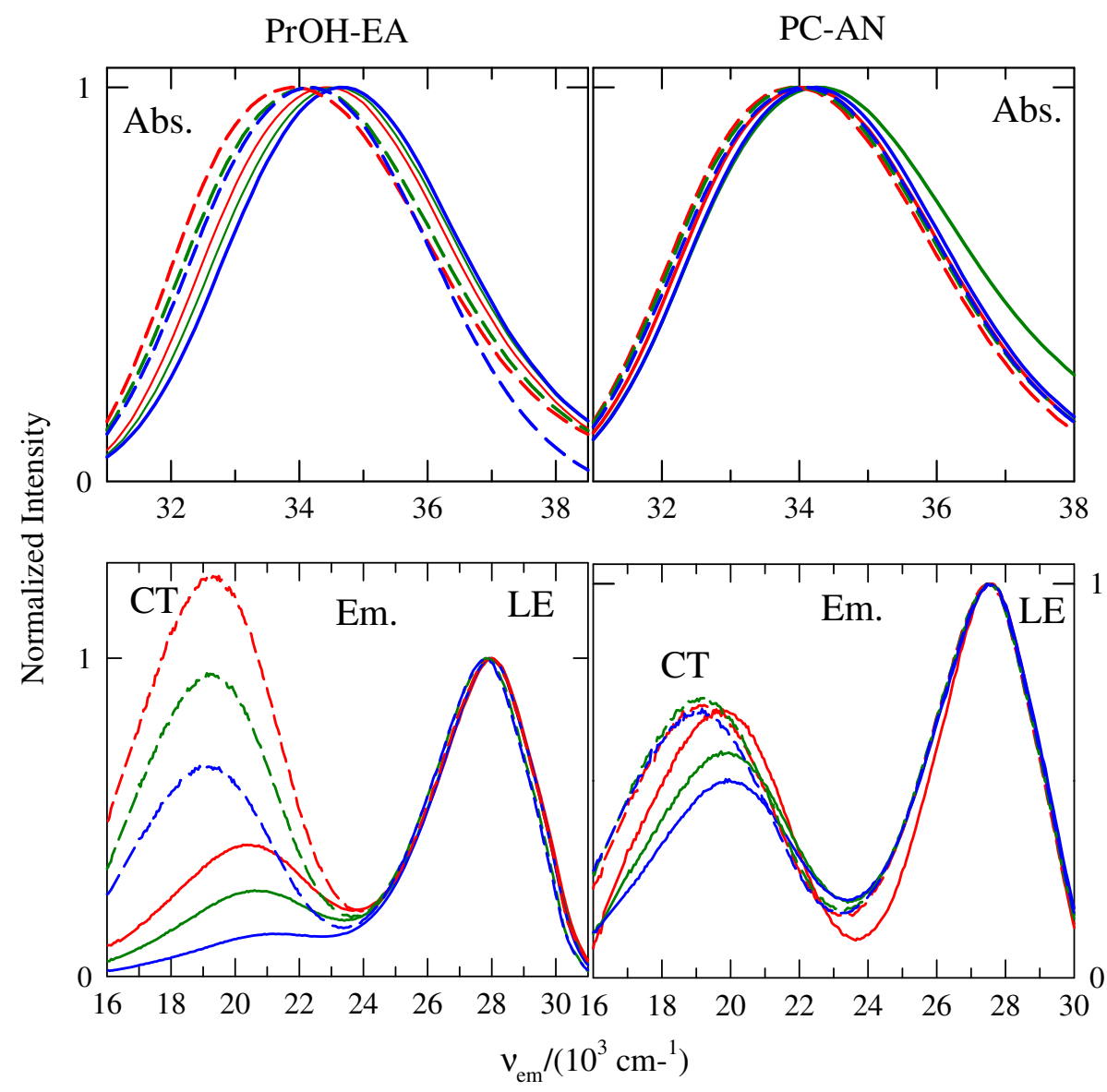

Figure 3. Absorption (upper panels) and emission spectra (lower panels) of $\mathrm{P} 4 \mathrm{C}$ in $(\mathrm{PrOH}+\mathrm{EA})$ and $(\mathrm{PC}+\mathrm{AN})$ mixed solvent systems at 0.2 (red), 0.5 (green) and 0.8 (blue) mole fraction of less polar solvent component (EA and AN). Legends at the top of the panels indicate the identity of the mixed solvent systems. Solid lines represent spectra without electrolyte and dashed lines represent spectra with electrolyte. Note that the electrolyte used is $\mathrm{LiClO}_{4}$ and its concentration in $(\mathrm{PrOH}+\mathrm{EA})$ mixture is $1.0 \mathrm{M}$ and in $(\mathrm{PC}+\mathrm{AN}) 0.5 \mathrm{M}$.

In weakly polar medium, smaller Debye screening length ${ }^{25}$ allows greater ion-solute interaction, producing a stronger electrolyte effects on spectral properties in weakly dipolar media. This is probably the reason for the observed much larger electrolyte-induced stabilization of the CT emission frequency in the weakly polar mixed solvent systems than in the relatively stronger counter-parts. The insensitivity of the CT emission frequency to the mole fraction of EA in presence of electrolyte in $(\mathrm{PrOH}+\mathrm{EA})$ mixtures may be explained as follows. EA, being the solvent with the lowest $\varepsilon_{0}$ value, facilitates the interaction between the existing ions and the solute. The ion-solute interaction in this medium is so favourable that successive addition of more polar $\mathrm{PrOH}$ cannot alter the composition of the solvation shell around the solute by displacing ions from it. This means that the preferential solvation of a dipolar solute in a mixture becomes secondary in presence of a more favourable ion-solute interaction. In fact, this is expected on the consideration that ion-solute (iondipole) interaction is stronger than the solute-solvent dipolar interactions involved in preferential solvation.

We present in figure 5 the mixture composition dependence of the ratio of areas under the CT and LE bands $\left(\alpha_{C T} / \alpha_{L E}\right)$ and the change in reaction free energy $\left(-\Delta G_{r}\right)$ for the $\mathrm{LE} \rightarrow \mathrm{CT}$ conversion reaction of $\mathrm{P} 4 \mathrm{C}$ in these mixed solvent systems in the absence and presence of electrolyte. While the dependence of $\alpha_{C T} / \alpha_{L E}$ on the mole fraction of the less polar solvent component (EA or AN) constituting these mixed solvent systems are shown in the left panels of this figure, the composition dependence of $-\Delta G_{r}$ is presented in the right panels. The change in reaction free energy has been calculated from the following 


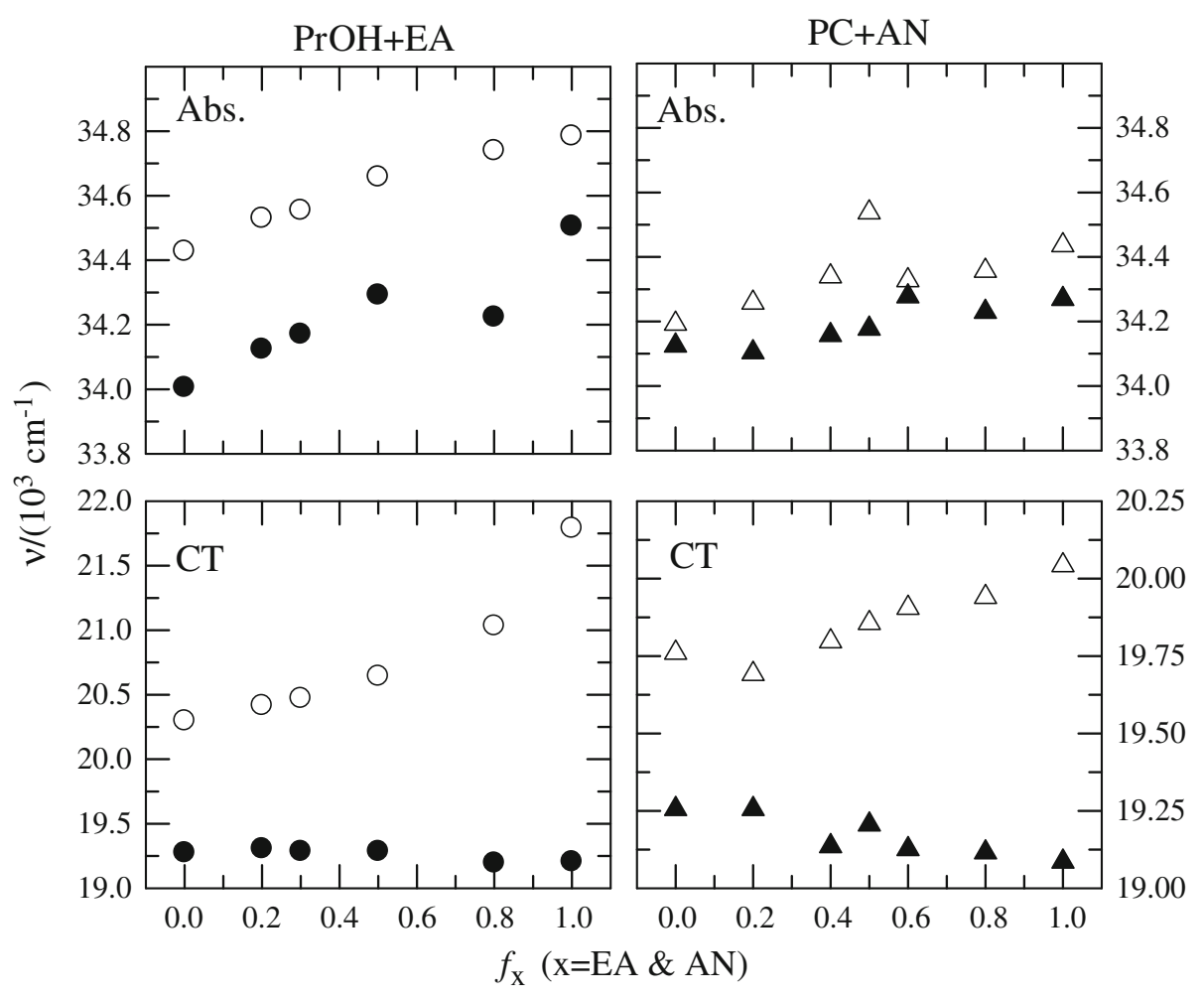

Figure 4. Mixture composition dependence of spectral (absorption and CT emission) peak frequencies of $\mathrm{P} 4 \mathrm{C}$ in $(\mathrm{PrOH}+\mathrm{EA})$ and $(\mathrm{PC}+\mathrm{AN})$ mixed solvent systems in absence (open symbols) and presence of electrolyte (filled symbols).

relation: ${ }^{21}-\Delta G_{r}=R T \ln K_{e q}=R T \ln \left(\alpha_{C T} v_{L E}^{3} /\right.$ $\alpha_{L E} v_{C T}^{3}$ ), where $K_{e q}$ denotes the equilibrium constant for the reaction and $R T$ the universal gas constant times the absolute temperature. Note for both the mixtures in the absence of electrolyte $\alpha_{C T} / \alpha_{L E}$ decreases almost linearly with the increase in mole fraction of the less polar solvent component. Interestingly, the decrease in $\alpha_{C T} / \alpha_{L E}$ for changing the solvent from PrOH to EA is more than an order of magnitude eventhough $\varepsilon_{0}$ values of these two solvents differ by just a factor of $\sim 3$. The decrease in $\alpha_{C T} / \alpha_{L E}$ is only by a factor of $\sim 1.6$ for changing the solvent from PC to AN, which correlates well with $\sim 1.8$ times reduction in $\varepsilon_{0}$ value of $\mathrm{AN}$ relative to that of PC. A much stronger reduction than expected for $\alpha_{C T} / \alpha_{L E}$ in EA occurs due to the reduction in solvent polarity and narrowing of the CT emission band in this solvent. Composition dependent CT emission bandwidths (full width at half maximum) summarized in table 2 indicate that while the $\mathrm{CT}$ emission band narrows down by $\sim 700 \mathrm{~cm}^{-1}$ for changing the solvent from $\mathrm{PrOH}$ to EA, bandwidth increases by $350 \mathrm{~cm}^{-1}$ if PC is replaced by AN. While the increase in CT bandwidth upon addition of alcohol in (EA + ProH) mixture may be attributed to the formation of H-bonding between the solute in its $\mathrm{CT}$ state and the surrounding
PrOH molecules, ${ }^{47}$ similar mechanism is surely not operative in $(\mathrm{PC}+\mathrm{AN})$ mixtures. Moreover, earlier studies with $\mathrm{P} 4 \mathrm{C}$ and its higher analogues in pure protic and aprotic solvents did not indicate presence of specific solute-solvent interactions in the steady state fluorescence aspects of these solutes although complicated fluorescence decay dynamics (that is, presence of a third component) in alcohols was hinted. ${ }^{21}$ In contrast, decay dynamics of $\mathrm{P} 4 \mathrm{C}$ in acetonitrile was found to be purely bi-exponential ${ }^{21}$ as has been observed here. Therefore, time-resolved infrared absorption experiments with $\mathrm{P} 4 \mathrm{C}$ in acetonitrile, as done in ref. 47 for 4dimethylaminobenzonitrile, may be carried out to ascertain the origin for the observed increase in bandwidth for changing the medium gradually from PC to AN.

Addition of electrolyte facilitates the formation of CT population in both the above mixed solvent systems, particularly in the relatively less polar solventrich region but the enhancement (of formation of CT) is much stronger for $(\mathrm{PrOH}+\mathrm{EA})$ mixtures. Again, more than an order of magnitude increase in $\alpha_{C T} / \alpha_{L E}$ is observed on addition of electrolyte in EA which is far greater than merely an increase in area ratio by approximately 1.6 times on addition of electrolyte in AN. This further supports the notion that ion-solute 


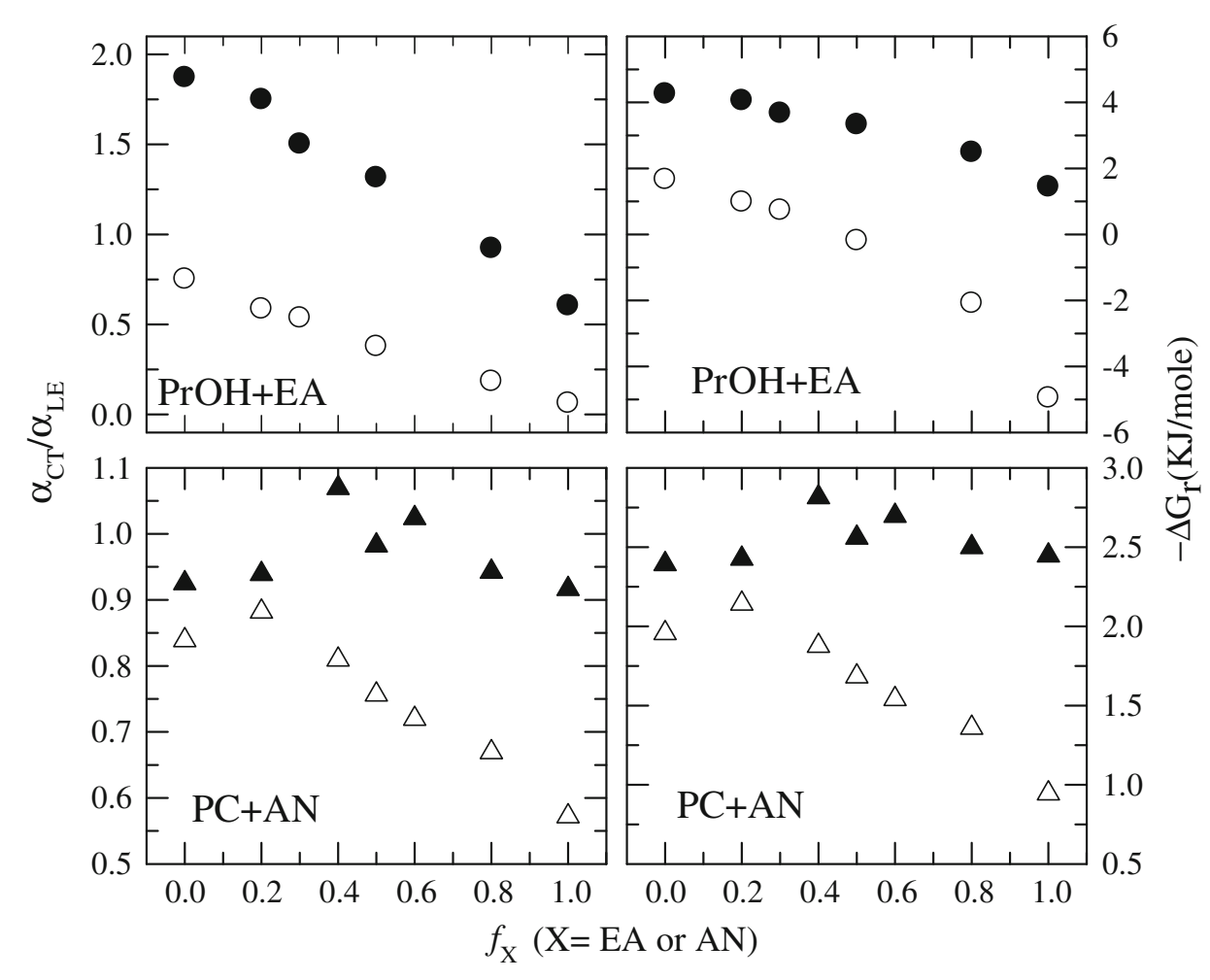

Figure 5. Composition dependences of the area ratio $\left(\alpha_{C T} / \alpha_{L E}\right)$ under the CT and LE emission bands and the reaction driving force $\left(-\Delta G_{r}\right)$ for the $\mathrm{LE} \rightarrow \mathrm{CT}$ conversion reaction of $\mathrm{P} 4 \mathrm{C}$ in $(\mathrm{PrOH}+\mathrm{EA})$ and $(\mathrm{PC}+\mathrm{AN})$ mixed solvent systems. While data in electrolyte solutions are represented by solid symbols, those in absence of electrolyte are denoted by the open ones. The uncertainty for band area ratio is typically within $\pm 10 \%$. Mixture-identities are indicated.

interaction is more pronounced in weakly polar solvent. Another interesting aspect is that $\alpha_{C T} / \alpha_{L E}$ in (PC + AN) mixtures remains almost insensitive to the mixture composition in presence of electrolyte. This is probably due to relatively larger solute-ion interaction in $(\mathrm{PC}+\mathrm{AN})$ mixtures at the AN-rich regime and this enhanced solute-ion interaction is also reflected in the gradual increase in the $\mathrm{CT}$ emission bandwidth (see table 2) in presence of electrolyte on successive addition of less polar solvent, AN. The change in CT bandwidth in presence of electrolyte is non-monotonic, decreasing with EA mole fraction in $\mathrm{PrOH}$ rich regime and then increasing as EA dominates the mixture composition (see table 2). Since $\mathrm{Li}^{+}$ion is believed to be a solution structure-maker (through the formation of solventberg), ${ }^{48,49}$ one expects an increase in bandwidth in the alcohol-rich regime. The reverse is observed probably because of the gradual increase in solute-ion interaction as EA is progressively added in the solution in presence of electrolyte. Since spectral width is determined by the solution polarity and heterogeneity, the observed non-monotonic EA mole fraction dependence can be attributed mainly to the decrease in solution polarity upon successive addition of EA up to 50:50 composition of the mixture and subsequently to the enhanced ion-solute interaction in the EA-rich mixtures. Note also that in the absence of electrolyte CT bandwidths are larger in $(\mathrm{PrOH}+\mathrm{EA})$ mixtures than in $(\mathrm{PC}+\mathrm{AN})$ mixtures and also shows an opposite solution polarity dependence. This and the width variation in presence of electrolyte strongly suggest that these two mixed solvent systems differ in interactions with a dissolved solute, supporting the conclusions made earlier based on the mixture composition dependent sound velocity and density measurements of these mixed solvent systems.

The mixture composition dependent change in reaction free energy or reaction driving force $\left(-\Delta G_{r}\right)$, shown in the right panels of figure 5 , reveals several important aspects. For example, the reaction driving force changes sign while the mixture converts from PrOH-rich to EA-rich solutions. This means that the reaction is becoming successively unfavourable upon continuous increase of EA in this mixture. In fact, the reaction equilibrium constant decreases from a value of $\sim 2.25$ in pure $\mathrm{PrOH}$ to $\sim 0.14$ in pure EA, implying 
Table 2. Spectral widths of LE and CT emission bands of P4C in mixed solvent systems both in presence and absence of $\mathrm{LiClO}_{4}$.

\begin{tabular}{cccccc}
\multicolumn{2}{l}{ A. PrOH + EA mixture } \\
\cline { 5 - 6 } $\mathrm{f}_{\mathrm{EA}}$ & \multicolumn{2}{c}{ Without $\mathrm{LiClO}_{4}$} & & \multicolumn{2}{c}{ With $1.0 \mathrm{M} \mathrm{LiClO}_{4}$} \\
\cline { 5 - 6 } \cline { 5 - 6 } & $\Gamma_{\mathrm{LE}}\left(10^{3} \mathrm{~cm}^{-1}\right)$ & $\Gamma_{\mathrm{CT}}\left(10^{3} \mathrm{~cm}^{-1}\right)$ & & $\Gamma_{\mathrm{LE}}\left(10^{3} \mathrm{~cm}^{-1}\right)$ & $\Gamma_{\mathrm{CT}}\left(10^{3} \mathrm{~cm}^{-1}\right)$ \\
\hline 0 & 1.44 & 4.22 & & 1.31 & 3.83 \\
0.2 & 1.31 & 3.93 & & 1.30 & 3.74 \\
0.3 & 1.30 & 3.90 & & 1.14 & 3.62 \\
0.5 & 1.24 & 3.85 & & 1.18 & 3.63 \\
0.8 & 1.15 & 3.86 & & 1.16 & 3.66 \\
1 & 1.13 & 3.51 & 1.17 & 4.03 \\
\hline
\end{tabular}

B. PC-AN mixture

\begin{tabular}{cccccc}
\hline $\mathrm{f}_{\mathrm{ACN}}$ & \multicolumn{2}{c}{ Without $\mathrm{LiClO}_{4}$} & & \multicolumn{2}{c}{ With $0.5 \mathrm{M} \mathrm{LiClO}_{4}$} \\
\cline { 5 - 6 } \cline { 5 - 5 } & $\Gamma_{\mathrm{LE}}\left(10^{3} \mathrm{~cm}^{-1}\right)$ & $\Gamma_{\mathrm{CT}}\left(10^{3} \mathrm{~cm}^{-1}\right)$ & & $\Gamma_{\mathrm{LE}}\left(10^{3} \mathrm{~cm}^{-1}\right)$ & $\Gamma_{\mathrm{CT}}\left(10^{3} \mathrm{~cm}^{-1}\right)$ \\
\hline 0 & 1.12 & 3.22 & & 0.96 & 3.18 \\
0.2 & 0.54 & 2.50 & & 1.06 & 3.32 \\
0.4 & 0.94 & 3.02 & & 0.65 & 2.94 \\
0.5 & 1.13 & 3.24 & & 1.16 & 3.55 \\
0.6 & 1.18 & 3.29 & & 0.97 & 3.25 \\
0.8 & 1.23 & 3.41 & 1.19 & 3.64 \\
1 & 1.34 & 3.57 & 1.29 & 3.82 \\
\hline
\end{tabular}

the $\mathrm{LE} \rightarrow \mathrm{CT}$ conversion reaction becoming $\sim 17$ times less favourable in EA compared to $\mathrm{PrOH}$. Moreover, addition of electrolyte in EA enhances $K_{e q}$ by a factor of $\sim 15$ over the value in the absence of electrolyte. In pure $\mathrm{PrOH}$, however, addition of electrolyte increases $K_{e q}$ only by $\sim 2.5$ times, again indicating more pronounced solute-ion interaction in the relatively less polar solvent. The enhanced solute-ion interaction in less polar solvent is further evident in the fact that in presence of electrolyte $K_{e q}$ decreases by a factor of $\sim 2.75$ in replacing $\mathrm{PrOH}$ by EA, which is a very moderate decrease compared to that (a factor of $\sim 17$ ) in the absence of electrolyte. In (PC $+\mathrm{AN})$ mixtures, the reaction is highly favoured as $-\Delta G_{r}$ remains positive at all mixture compositions. Here the decrease in $K_{e q}$ is just by a factor of $\sim 1.5$ for interchanging the solvent component. Note that while addition of electrolyte in PC increases $K_{e q}$ by merely 1.2 times over the pure solvent value, the increase in electrolyte solution of AN is by a factor of $\sim 2$. This again indicates stronger ionsolute interaction in $\mathrm{AN}$ which is relatively less polar than PC.

\subsection{Time resolved studies}

Time resolved emission decays of $\mathrm{P} 4 \mathrm{C}$ in these binary mixtures both in presence and absence of $\mathrm{LiClO}_{4}$ have been found to be adequately described by biexponential functions of time with a fast and a slow time constants. Representative fluorescence emission intensity decays (LE and CT) along with the bi-exponential fits and the residuals are shown in figure 6 for $(\mathrm{PrOH}+$ EA) mixture at 50:50 mole ratio in the absence of any electrolyte and in presence of $1.0 \mathrm{M} \mathrm{LiClO}_{4}$. The instrument response function and the fit parameters are also shown in order to stress the adequacy of bi-exponential fit functions. Similar fits for intensity decays of $\mathrm{P} 4 \mathrm{C}$ in $(\mathrm{PC}+\mathrm{AN})$ mixture at 0.5 mole fraction of $\mathrm{ACN}$ in the absence and presence electrolyte are shown in supporting information (figure S1). Note that both LE and CT emission decays fit adequately with bi-exponential functions, producing CT rise time constants similar to the fast time constants found in LE emission decays. Therefore, the short time constant is ascribed to the reaction time (that is, $\tau_{1} \equiv \tau_{r x n}$ ) whereas the long time constant is assumed to be the long life time (that is, $\tau_{2} \equiv \tau_{\text {long }}$ ) of the states involved. ${ }^{21}$ The goodness of fit parameter $\left(\chi^{2}\right)$ for these and other mixtures being nearly unity and the absence of any regular pattern in the residuals strongly suggest the reaction kinetics of $\mathrm{P} 4 \mathrm{C}$ in these mixed solvent systems conform to the classical two state reversible reaction mechanism as observed earlier in different reaction environments. ${ }^{21-28}$ Interestingly, addition of electrolyte both shortens the reaction time constant $\left(\tau_{1}\right)$ 

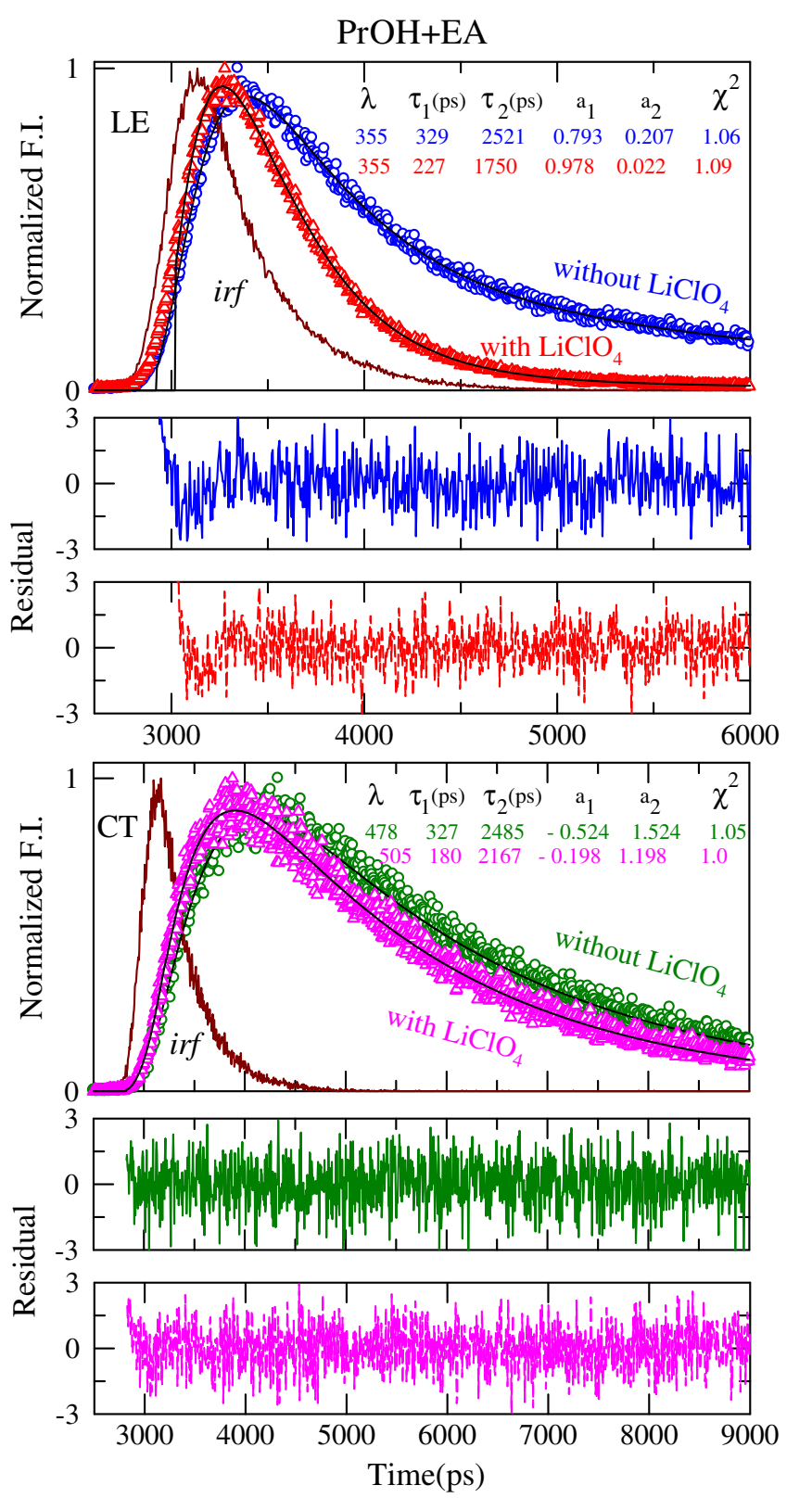

Figure 6. Fluorescence LE (upper panels) and CT (lower panel) emission intensity decays of $\mathrm{P} 4 \mathrm{C}$ in (PrOH $+\mathrm{EA})$ mixture at 0.5 mole fraction of ethyl acetate both in presence and absence of $1.0 \mathrm{M} \mathrm{LiClO}_{4}$. Bi-exponential fit parameters, instrument response function ('irf') and the residuals at are shown. While the blue and red circles represent data of LE intensity decay respectively in absence and presence of electrolyte, green and pink circles denote data of CT intensity decay in absence and presence of electrolyte. The residuals shown below the respective decays are also tagged with the same colour code.

and enhances the amplitude $\left(a_{1}\right)$ significantly at this composition of $(\mathrm{PrOH}+\mathrm{EA})$ mixture. Moreover, the ratio of the equilibrium constants in the presence and absence of electrolyte determined from the LE decay amplitudes $\left(K_{e q}^{\text {time-resolved }}=a_{1} / a_{2}\right)$ is $\sim 16$ which is in close agreement with the value $(\sim 15)$ obtained from the steady state measurements.

Next the mixture composition dependent reaction time and long time constants are shown in figure 7 where $\tau_{r x n}$ and $\tau_{\text {long }}$ for both (PrOH + EA) and (PC + AN) mixed solvent systems in presence and absence of $\mathrm{LiClO}_{4}$ are presented as a function of mole fraction of the relatively less polar solvent component (EA or AN) constituting the mixtures. Analogous time constants from LE decays for these systems are shown in figure S2 (supporting information). Time constants presented in these figures reflect that while in electrolyte solutions of $(\mathrm{PrOH}+\mathrm{EA})$ mixture both the $\tau_{r x n}$ and $\tau_{\text {long }}$ values are shorter than those measured in the absence of electrolyte, $\tau_{r x n}$ in $(\mathrm{PC}+\mathrm{AN})$ mixture becomes insensitive to the presence of electrolyte at all mole fractions of AN eventhough $\tau_{\text {long }}$ becomes shorter. What is even more interesting is that $\tau_{r x n}$ in $(\mathrm{PC}+\mathrm{AN})$ mixed solvent systems is independent of the mixture composition in the absence of electrolyte. The mixture composition dependence of $\tau_{r x n}$ in $(\mathrm{PrOH}+$ EA) mixtures and independence of it in $(\mathrm{PC}+\mathrm{AN})$ in the absence and presence of electrolyte are completely new results and require further study to better understand this phenomenon. At the present moment, however, the composition dependence of $\tau_{r x n}$ in these two mixed solvent systems may be qualitatively explained as follows. If the intramolecular charge transfer process in $\mathrm{P} 4 \mathrm{C}$ molecule can be regarded as 'electron transfer reaction' then the following relation ${ }^{50-54}$ connects the reaction rate to the activation energy $\left(\Delta G^{*}\right)$ and average solvation time $\left(\left\langle\tau_{s}\right\rangle\right)$ in these mixed solvent systems: $\tau_{r x n}^{-1} \equiv k_{E T}=\left\langle\tau_{s}\right\rangle^{-1} \exp \left[-\Delta G^{*} / R T\right]$ with $\Delta G^{*}=$ $(\lambda / 4)\left[1-\left(-\Delta G_{r} / \lambda\right)\right]^{2}$, where $-\Delta G_{r}$ and $\lambda$ denote respectively the reaction driving force and the total reorganization energy. Note that ${ }^{55} \lambda \approx \lambda_{\text {sol }}=\Delta v^{t} / 2 \approx$ $\Delta \Delta v / 2$, where $\Delta v^{t}$ is the dynamic Stokes' shift measured with a polarity probe in solvation dynamics experiments and $\Delta \Delta v$ the steady state Stokes' shift for the same polarity probe relative to a non-polar solvent. $\lambda_{\text {sol }}$ in these pure solvents ${ }^{32}$ is in the range of $\sim 1000 \mathrm{~cm}^{-1}$ and it has already been shown that $\lambda_{\text {sol }}$ remains insensitive to the mixture composition for ( $\mathrm{PC}+\mathrm{AN}$ ) mixed solvent system. ${ }^{33}$ This mixture composition insensitivity of $\lambda_{\text {sol }}$ is also preserved in presence of $0.5 \mathrm{M}$ $\mathrm{LiClO}_{4}$ in $(\mathrm{PC}+\mathrm{AN})$ mixtures at all AN mole fractions (see figure $\mathrm{S} 3$, supporting information). $-\Delta G_{r}$, shown already in figure 5 , suggests a moderate composition dependence for $(\mathrm{PC}+\mathrm{AN})$ mixture but becomes independent of AN mole fraction in presence of electrolyte. Since earlier studies ${ }^{21}$ have indicated either no 

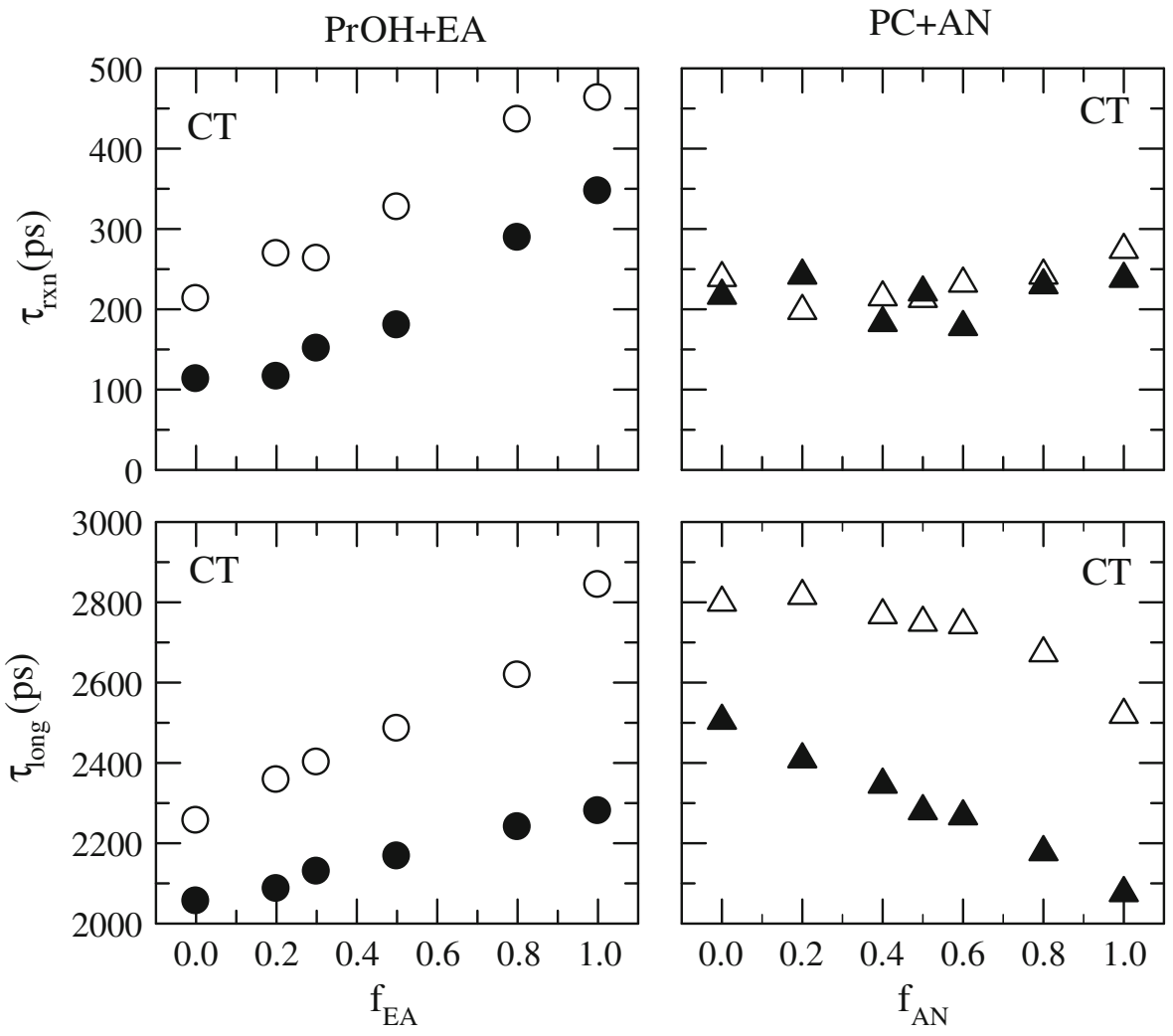

Figure 7. Composition dependence of the reaction time ( $\tau_{r \times n}$, upper panels) and long time $\left(\tau_{\text {long }}\right.$, lower panels) constants obtained from bi-exponential fit of the collected CT emission decays of $\mathrm{P} 4 \mathrm{C}$ in $(\mathrm{PrOH}+\mathrm{EA})$ and $(\mathrm{PC}+\mathrm{AN})$ mixed solvent systems in the presence (filled symbols) and absence (open symbols) of electrolyte. The uncertainty associated with these time constants is within $\pm 5 \%$ of the reported values.

or insignificant dynamic solvent control of $\tau_{r x n}$ in P4C (in other words, insensitivity to $\left\langle\tau_{s}\right\rangle$ ), it can now be understood the observed mixture composition independence of $\tau_{r x n}$ for $\mathrm{P} 4 \mathrm{C}$ in $(\mathrm{PC}+\mathrm{AN})$ mixtures both in the presence and absence of the electrolyte. In $(\mathrm{PrOH}+$ EA) mixtures, $-\Delta G_{r}$ decreases quite significantly with the increase in EA mole fraction. In addition, $-\Delta G_{r}$ becomes larger in presence of electrolyte at all mole fractions of EA. $\Delta \Delta v$, shown in figure $\mathrm{S} 3$, indicate a small difference between the values in the absence and presence of electrolyte in (PrOH + EA) mixed solvent systems. This explains the observed much stronger mixture composition dependence of $\tau_{r x n}$ and shortening of it in presence of electrolyte in the weakly polar $(\mathrm{PrOH}+\mathrm{EA})$ mixed solvent systems.

\section{Conclusion}

Let us first summarize the main results of this paper. The dependence on mixture composition of the
$\mathrm{LE} \rightarrow \mathrm{CT}$ conversion reaction has been explored in two mixed solvent systems of widely separated polarities. Density and sound velocity measurements have indicated that the low polar ( $\mathrm{PrOH}+\mathrm{EA})$ mixed solvent systems are structurally different from the strongly polar $(\mathrm{PC}+\mathrm{AN})$ mixtures. Steady state spectroscopic studies in these mixtures and their electrolyte solutions have revealed that electrolyte effects are much stronger in the low polarity mixed solvent systems than in the strongly polar counterpart. The CT emission band frequency (peak) in electrolyte solutions of ( $\mathrm{PrOH}+\mathrm{EA})$ mixture has been found to be insensitive to the EA concentration in the mixture, indicating strong ion-solute interaction in low polar solutions. Changes in reaction free energy show a strong electrolyte effects and composition dependence for low polar systems but a moderate dependence in high polar mixtures which becomes mixture composition independent in the latter system in presence of electrolyte. The reaction time constant also shows strong mole fraction dependence for the low polar mixture and addition of electrolyte has been found 
to considerably enhance the reaction rate. In the high polar mixture, however, the reaction time constant neither senses the effects of electrolyte nor depends on the mixture composition. These observations have been qualitatively explained in terms of the solvent reorganization energy and reaction driving force by using the classical theory of electron transfer reaction. It would be interesting to expand this study for those mixed solvent systems which show pronounced solution heterogeneity with mixture composition and temperature, ${ }^{56,57}$ and where addition of salt may induce appearance of a closed-loop two-phase region in the phase diagram of the binary mixture. ${ }^{58,59}$

\section{Supplementary information}

The table S1 and figures S1, S2 and S3 are given as supplementary information (see www.ias.ac.in/chemsci).

\section{Acknowledgements}

We thank Prof. M Maroncelli for support and encouragement. HG thanks the Vice Chancellor, Aliah University, West Bengal for encouragement.

\section{References}

1. Zerres H and Prasunitz J M 1994 Thermodynamics 40 676

2. Mock B, Evans L B and Chen C C 1986 AIChE J. 32 1655

3. Kolker A and de Pablo J 1996 Ind. Eng. Chem. Res. 35 234

4. Faverio C L, Mussini P R and Mussini T 1998 Anal. Chem. 702589

5. Wang P, Aderko A and Young R D 2002 Fluid Phase Equilib. 203141

6. Schmitt D and Vogelpohl A 1982 Fluid Phase Equilib. 9 167

7. Hala E 1983 Fluid Phase Equilib. 13311

8. Maurer G 1983 Fluid Phase Equilib. 13269

9. Sander B, Fredenslund A and Rasmussen P 1986 Chem. Eng. Sci. 411171

10. Rousseau R W, Ashcraft D L and Schoenborn E M 1972 AIChE J. 18825

11. Kamps A P S 2005 Ind. Eng. Chem. Res. 44201

12. Takamuku T, Tsutsumi Y, Matsugami M and Yamaguchi 2008 J. Phys. Chem. B 11213300

13. Shi J, Chen J, Feng, Chen T, Lian Y, Wang X and Li C 2007 J. Phys. Chem. C 111693

14. Micali N, Trusso S, Vasi C, Blaudez D and Mallamace F 1996 Phys. Rev. E 541720

15. Wetzler D E, Chesta C, Fernandez-Prini R and Aramendia P F 2002 J. Phys. Chem. A 1062390
16. Hicks J, Vandersall M, Babarogic Z and Eisenthal K B 1985 Chem. Phys. Lett. 11618

17. Hicks J, Vandersall M, Babarogic Z and Eisenthal K B 1987 Chem. Phys. Lett. 135413

18. Nag A, Kundu T and Bhattacharyya K 1989 Chem. Phys. Lett. 160257

19. Hynes J T 1994 Charge - transfer reactions and solvation dynamics in ultrafast dynamics of chemical systems; J. D. Simon (Ed.) Dodrecht: Kluwer; p.345

20. van der Zwan G and Hynes J T 1991 Chem. Phys. 152 169

21. Dahl K, Biswas R, Ito N and Maroncelli M 2005 J. Phys. Chem. B 1091563

22. Pradhan T, Gazi H A R and Biswas R 2010 J. Chem. Sci. 122481

23. Pradhan, T and Biswas R 2007 J. Phys. Chem. A. 111 11524

24. Pradhan T and Biswas R 2009 J. Solution Chem. 38517

25. Pradhan T, Gazi H A R and Biswas R 2009 J. Chem. Phys. 131054507

26. Pradhan T and Biswas R 2007 J. Phys. Chem. A 111 11514

27. Pradhan T, Ghoshal P and Biswas R 2008 J. Phys. Chem. A 112915

28. Pradhan T, Ghoshal P and Biswas R 2009 J. Chem. Sci. 12195

29. Covington A K and Newman K E 1979 Pure Appl. Chem. 512041

30. Suppan P 1987 J. Chem. Soc. Faraday Trans. I 83495

31. Suppan P 1990 J. Photochem. Photobiol. A. Chem. 50 293

32. Horng M L, Gardecki J A, Papazyan A and Maroncelli M 1995 J. Phys. Chem. 9917311

33. Gardecki J A and Maroncelli M 1999 Chem. Phys. Lett. 301571

34. Reichardt C 1994 Chem. Rev. 942319

35. Kamlet M J, Abboud J L and Taft W R 1977 J. Amer. Chem. Soc. 986027

36. Laurence C, Nicolet P, Dalati M T, Abboud J L M and Notario R 1994 J. Phys. Chem. 985807

37. Yoon B J and Ohr Y G 2000 J. Chem. Phys. 1138149

38. Grabowski Z R, Rotkiewicz K and Rettig W 2003 Chem. Rev. 1033899

39. Lippert E, Rettig W, Bonacic-Koutecky V, Heisel F and Mieche J A 1987 Adv. Chem. Phys. 681

40. Zachariasse K A, Grobys M, von der Haar T, Hebecker A, Il'ichev Y V, Jiang Y B, Morawski O and Kuhnle W 1996 J. Photochem. Photobiol. A 10259

41. Zachariasse K A 2000 Chem. Phys. Lett. 3208

42. Rettig W 1980 J. Luminesc. 2621

43. Rettig W 1982 J. Phys. Chem. 861970

44. Biswas R, Lewis J E and Maroncelli M 1999 Chem. Phys. Lett. 310485

45. Maroncelli M and Fleming G R 1987 J. Chem. Phys. 86 6221

46. Chapman C F and Maroncelli M 1991 J. Phys. Chem. 95 9095

47. Kwok W-M, George M W, Grills D C, Ma C, Matousek P, Parker A W, Phillips D, Toner W T and Towrie M 2003 Angew. Chem. Int. Ed. 421826

48. Kay R L and Evans D F 1966 J. Phys. Chem. 702325 
49. Harned H S and Owen B B 1958 The physical chemistry of electrolyte solutions 3rd Ed. (New York: Reinhold)

50. Zusman L D 1980 Chem. Phys. 49295

51. Marcus R A 1964 Annu. Rev. Phys. Chem. 15155

52. Marcus R A 1965 J. Chem. Phys. 43379

53. Marcus R A 1965 J. Chem. Phys. 43679

54. Larson S L, Cooley L F, Elliot C M and Kelley D F 1992 J. Am. Chem. Soc. 1149504

55. van der Zwan G and Hynes J T 1985 J. Phys. Chem. 89 4181
56. Gazi H A R and Biswas R 2011 Heterogeneity in binary mixtures of (water + tertiary butanol): Temperature dependence across mixture composition J. Phys. Chem. A 1152447

57. Roy S, Banerjee S, Biyani N, Jana B and Bagchi B 2011 J. Phys. Chem. B 111685

58. Bender T M and Pecora R 1986 J. Phys. Chem. 90 1700

59. Euliss G and Sorensen C M 1984 J. Chem. Phys. 81 1908 